\title{
Transperitoneal Laparoscopic Pyeloplasty versus Laparoscopic Assisted Pyeloplasty for the Treatment of Ureteropelvic Junction Obstruction in Children ${ }^{1}$ A hmed Abdelmohsen, ${ }^{1}$ Rafik Shalaby, ${ }^{2}$ Hussein Galal, and ${ }^{1}$ Samir Gouda \\ ${ }^{1}$ Department of Pediatric Surgery and ${ }^{2}$ Department of Urology, Faculty of Medicine, Al-Azhar University Corresponding author: Ahmed Abdelmohsen Said, email: a.abdelmohsen@azhar.edu.eg, mobile: 00201011252126
}

\begin{abstract}
Background: Laparoscopy-assisted pyeloplasty [LAP] has been introduced to shorten the operative time and make the traditional transperitoneal laparoscopic pyeloplasty [LP] much easier while maintaining comparable functional and cosmetic results to the LP. Purpose: Comparing the outcomes of LP to LAP in terms of operative time and cosmetic appearance. Patients and Methods: This is a randomized controlled study where twenty patients presented with ureteropelvic junction obstruction [UPJO] were divided into two equal groups. The first was subjected to LP while the second was subjected to LAP. The outcome measurements included the operative time, the hospital stay, intraoperative complications, and the success rates. Postoperative data were collected with special emphasis on pain and analgesia requirements.
\end{abstract}

Results: The mean age of the patients was $4.95 \pm 3.7$ years [range $=9$ months to 12 years], the preoperative SFU grade was 3.7 and the renal relative function was $25.5 \%$. The mean operative time was $154 \pm 21$ minutes [range $=126$ to 185 minutes] for LP compared to just $114 \pm 12$ minutes [range $=98$ to 130 minutes] in LAP $(\mathrm{p}<0.001)$. The success rate was $90 \%$ in both groups and the postoperative split function rose to around $40 \%$. Although the leakage was slightly higher in the LP, the wound complications were higher in the LAP group. The cosmetic results and patients' satisfaction were in favor of LP.

Conclusion: The success and recurrence rates are almost equal in both techniques. The main advantages of LAP over LP are the marked operative time reduction and its reproducibility by the less experienced laparoscopic surgeons.

Keywords: Laparoscopy, laparoscopy-assisted, pyeloplasty, extracorporeal, UPJO.

\section{INTRODUCTION}

The growing experience with ablative and reconstructive urologic laparoscopic procedures has encouraged pediatric surgeons to attempt the laparoscopic approach to repair UPJO. Since it was first introduced in 1993, LP has achieved comparable results to that of open surgery, while achieving the added goals of low morbidity, shorter hospital stay and shorter convalescence. Excellent results have been reported no matter which method has been used even in difficult cases with atypical anatomical anomalies $^{(1)}$. For these reasons, LP was considered the treatment of choice for primary UPJO; however, the technical challenges associated with laparoscopy and intracorporeal suturing have limited its widespread use ${ }^{(2,3)}$.

Nevertheless, since the first application of LP in pediatrics by Peters et al..$^{(4)}$, the laparoscopic approach has not been as popular in pediatric urology as in the adult population, possibly due to its technical difficulty and long learning curve. During the last two decades various minimally invasive surgical techniques such as laparoscopy-assisted extracorporeal pyeloplasty (LAP), and robot-assisted laparoscopic pyeloplasty have been developed and popularized in clinical practice. LAP is a hybrid technique of conventional laparoscopic surgery and extracorporeal hand sewing anastomosis ${ }^{(5)}$.
To date, some reports including meta-analysis have demonstrated the efficacy and safety of minimally invasive pyeloplasty in the pediatric population $^{(6-8)}$. However, there have been few studies, directly comparing recent surgical techniques ${ }^{(5)}$. In the present study, we described the clinical characteristics and compared the surgical outcomes of LP and LAP for the treatment of UPJO in children.

\section{PATIENTS AND METHODS}

This is a randomized controlled study conducted at Al-Azhar University Hospitals, combined with some private hospitals under supervision of Al-Azhar's Pediatric Surgery and Urology Departments' staff between May 2017 and December 2019. A total of 20 patients with UPJO's were divided into two equal groups $[\mathrm{No}=10]$. Method of randomization used was the closed envelop method.

Group A was subjected to LP in which a total transperitoneal laparoscopic pyeloplasty was done. Group B was subjected to LAP in which the UPJ was exteriorized out the abdominal cavity through a $2-\mathrm{cm}$. flank incision, after UPJ was completely exposed laparoscopically, and subsequently the obstructed UPJ segment was excised, the proximal ureter was spatulated and then the anastomosis was done extracorporeally identical to the open technique using 5/0 Vicryl sutures (Figs. 1-3). All the patients' data 
were thoroughly evaluated with regards to history, clinical examination, routine laboratory investigations, and imaging workup, which included renal U/S with categorizing pelvic dilatation according to the Society for Fetal Urology (SFU) grading system ${ }^{(9)}$, and diuretic renography. Tc-99 mercaptoacetyltriglycine (MAG3) scan was performed to evaluate drainage, glomerular filtration rate (GFR), and split renal function (SRF). The degree of anatomical and functional improvement including pre- and postoperative split function of the kidneys and the degree of hydronephrosis on the SFU grading system were recorded to evaluate the success of each procedure. The pain was recorded daily according to Wong-Baker FACES Foundation's pain scale $^{(10)}$ and the WHO step ladder analgesia ${ }^{(11)}$ was used in all patients.

Patient's satisfaction about the cosmetic results was evaluated using a subjective score ranging from $1-5$.

\section{ETHICAL CONSIDERATIONS}

This study was approved by Al-Azhar University's ethical committee and an informed consent was take from all the parents of the patients.

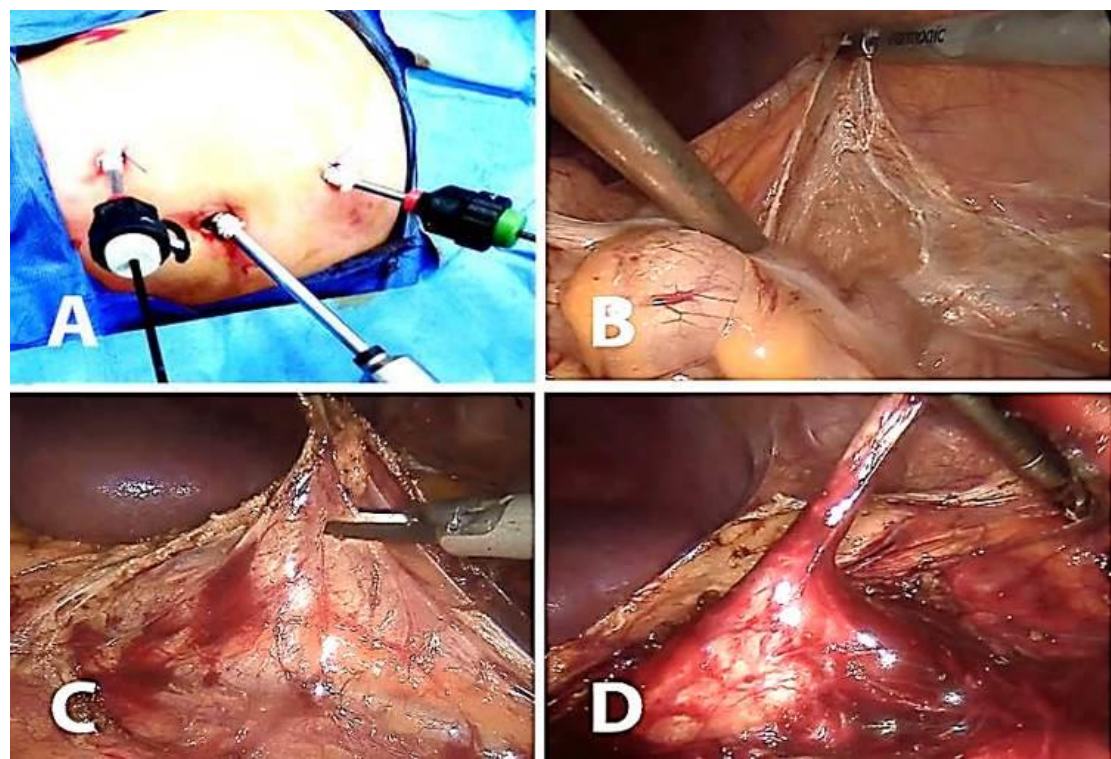

Figure 1: Illustrates the port sites and the steps of laparoscopic dissection of renal pelvis starting by 3 ports introduction (A). Mobilization of the colon to open the Gerota's fascia (B and C). A complete dissection of the ureteropelvic junction with the dilated pelvis (D).
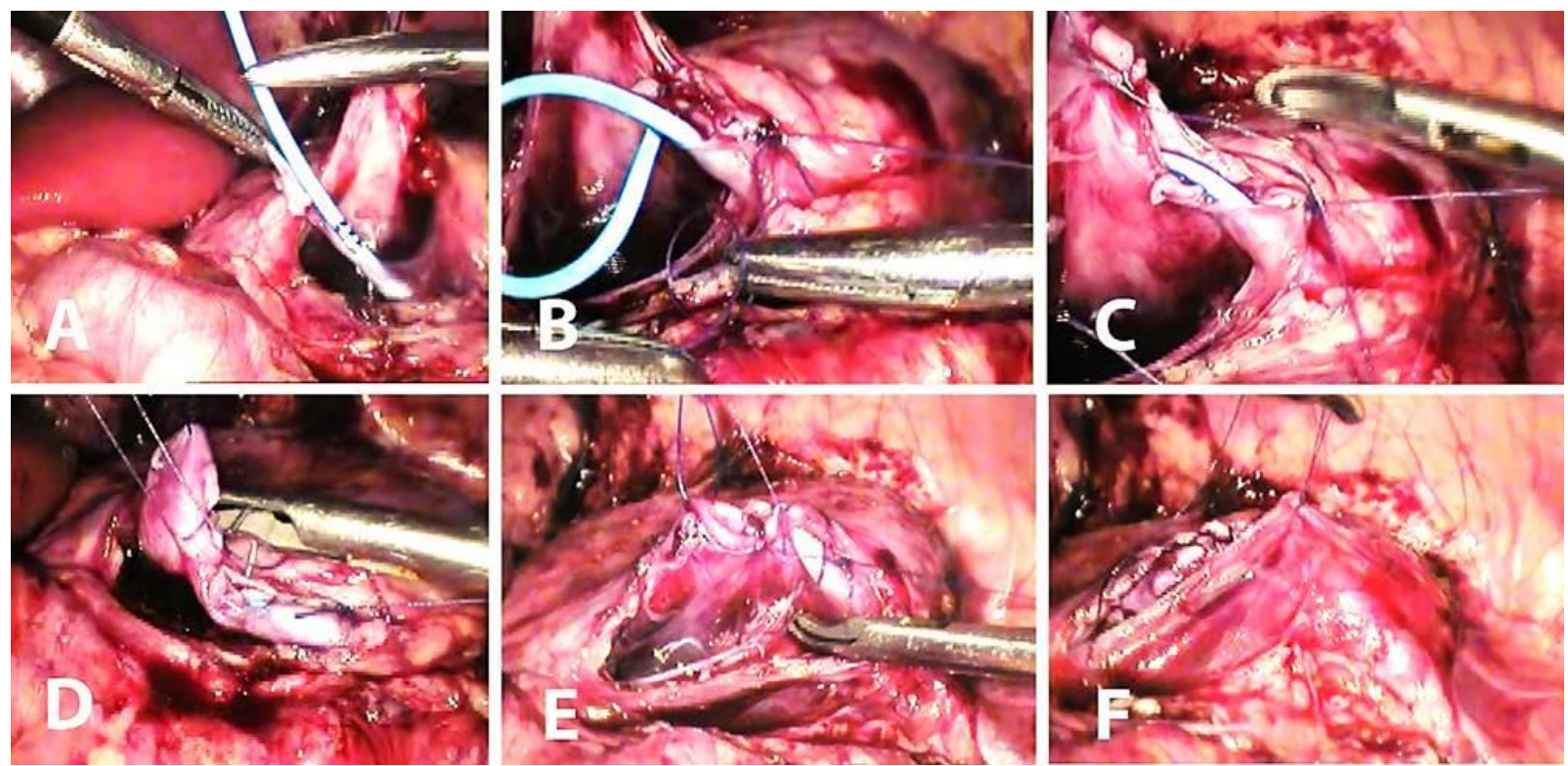

Figure 2: Illustrates the steps of LP including the insertion of the ureteric catheter in the ureteral end (A) suturing of the posterior wall first (B). Suturing of the anterior wall over the ureteral catheter (C, $\mathrm{D}$, and E.) Closure of renal fascia over the anastomosis (F). 

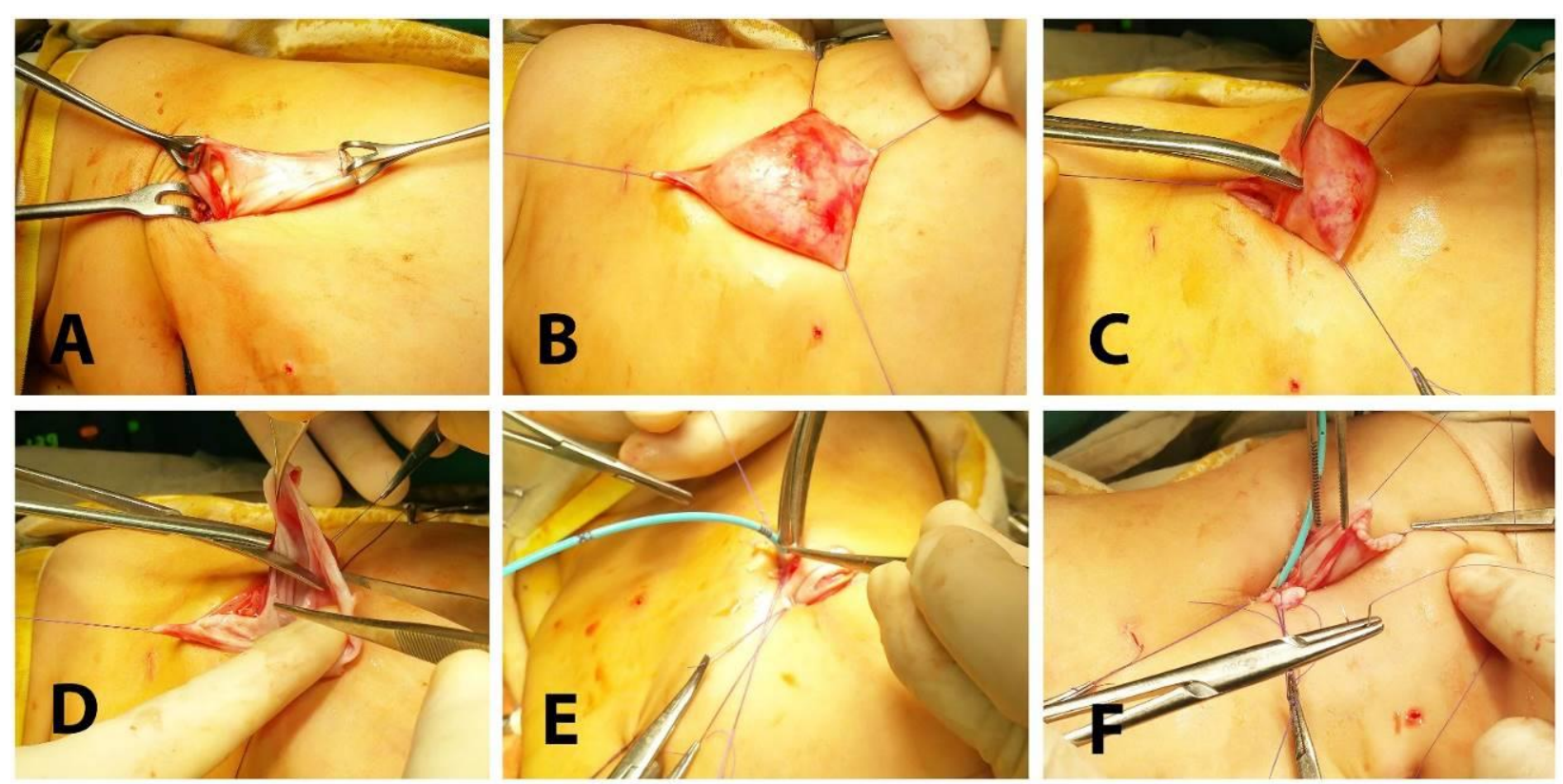

Figure 3: Illustrates the steps of the extracorporeal anastomosis including renal pelvis extraction from a flank wound (A and B). Cutting of the obstructed the segment and the dilated part of the renal pelvis $(\mathrm{C}$ and $\mathrm{D})$. Manual repair by hand sewing over a ureteric catheter $(\mathrm{E}$ and $\mathrm{F})$.

\section{Statistical Analysis}

The data were coded, entered and processed using IBM ${ }^{\circledR}$ SPSS ${ }^{\circledR}$ Statistics version 25. The numerical data were expressed as mean $\pm \mathrm{SD}$ and were compared by Student's t-test and categorical data were expressed in numbers and percentages and were compared by $\mathrm{Chi}^{2}$ test or Fisher's exact test as appropriate. $\mathrm{P}$ value was considered significant when it is less than 0.05 .

\section{RESULTS}

The patients were 14 males $(70 \%)$ and 6 females $(30 \%)$. Their mean age was $4.95 \pm 3.71$ years [Range $=9$ months to 12 years]. The demographic data of all patients are shown in table 1 .

Table 1: Demographic data of all patients.

\begin{tabular}{|c|c|c|c|c|c|c|c|c|c|c|}
\hline & & \multicolumn{8}{|c|}{ Groups } & \multirow{3}{*}{$\begin{array}{c}\text { P- } \\
\text { value }\end{array}$} \\
\hline & & \multicolumn{4}{|c|}{ Group A No=10 } & \multicolumn{4}{|c|}{ Group B No=10 } & \\
\hline & & Mean & SD & Number & $\%$ & Mean & SD & Number & $\%$ & \\
\hline \multicolumn{2}{|c|}{ Age in years } & 7.5 & 3.7 & & & 3.4 & 1 & & & $0.001 *$ \\
\hline \multirow{2}{*}{ Sex } & $\mathbf{M}$ & & & 6 & $60 \%$ & & & 8 & $80 \%$ & \multirow{2}{*}{$>0.05$} \\
\hline & $\mathbf{F}$ & & & 4 & $40 \%$ & & & 2 & $20 \%$ & \\
\hline \multirow{2}{*}{$\begin{array}{l}\text { Affected } \\
\text { Kidney }\end{array}$} & Right & & & 3 & $30 \%$ & & & 6 & $60 \%$ & \multirow{2}{*}{$>0.05$} \\
\hline & Left & & & 7 & $70 \%$ & & & 4 & $40 \%$ & \\
\hline \multirow{4}{*}{ Presentation } & $\begin{array}{l}\text { Prenatal } \\
\text { Diagnosis }\end{array}$ & & & 0 & $0 \%$ & & & 4 & $40 \%$ & \multirow{4}{*}{$<0.01$} \\
\hline & $\begin{array}{l}\text { Abdominal } \\
\text { Pain }\end{array}$ & & & 8 & $80 \%$ & & & 1 & $10 \%$ & \\
\hline & Flank swelling & & & 0 & $0 \%$ & & & 2 & $20 \%$ & \\
\hline & Incidental & & & 2 & $20 \%$ & & & 3 & $30 \%$ & \\
\hline \multirow{2}{*}{ UTI $* *$} & Yes & & & 2 & $20 \%$ & & & 1 & $10 \%$ & \multirow{2}{*}{$>0.05$} \\
\hline & No & & & 8 & $80 \%$ & & & 9 & $90 \%$ & \\
\hline
\end{tabular}

$* \mathrm{P}$-value less than 0.01 is highly significant. $\mathrm{UTI}^{* *}=$ Urinary tract infection 
The range of operative time in the LP group was 129 to 185 minutes compared to 95 to 130 minutes in the LAP group. The range of drain output in the LP group was 80 to $350 \mathrm{ml}$ while it was 10 to $140 \mathrm{ml}$ in the LAP group. There was no significant blood loss in either of the groups (Table 2).

Only a 10 -year-old female child $(10 \%)$ with completely intrarenal pelvis was converted to open surgery in the LP group. The success ratio in each group was $90 \%$. There were 2 patients, one $(10 \%)$ in each group, suffered from a recurrence of the flank pain, with radiological evidence of obstruction at the new UPJ. Only two patients in the LAP group suffered from wound problems, one of them had just a seroma of the wound and it was aspirated twice before complete resolution and the other patient had infection in the wound that was treated conservatively. Only, one patient $(10 \%)$ had urine leakage after LP and was managed conservatively and the leakage ceased after a week without urinoma.

Preoperative SFU grade was grade 4 in around $75 \%$ of all the patients, and the remaining $25 \%$ were grade 3 with almost no difference between the two groups; however, there were statistically insignificant lower SFU grads in the LAP group than the LP's (table 3).

Preoperative range of split function in the LP group was $15 \%$ to $38 \%$ compared to $10 \%$ to $37 \%$ in the LAP group. Both groups showed an improvement to $40.2 \pm 18.7$ [range $=25 \%$ to $66 \%$ ] and $41.7 \pm 13.3$ [range $=30 \%$ to $56 \%$ ] respectively (Table 4 ).

The cosmetic results (Figs. 4-5) were obviously better in the LP group than the LAP group since $80 \%$ of the patient's parents rated score 5, while the remaining $20 \%$ rated score 4 . By comparison, $40 \%$ of the patients rated score $4,40 \%$ rated score 3 and $20 \%$ rated score 2 in the LAP group.

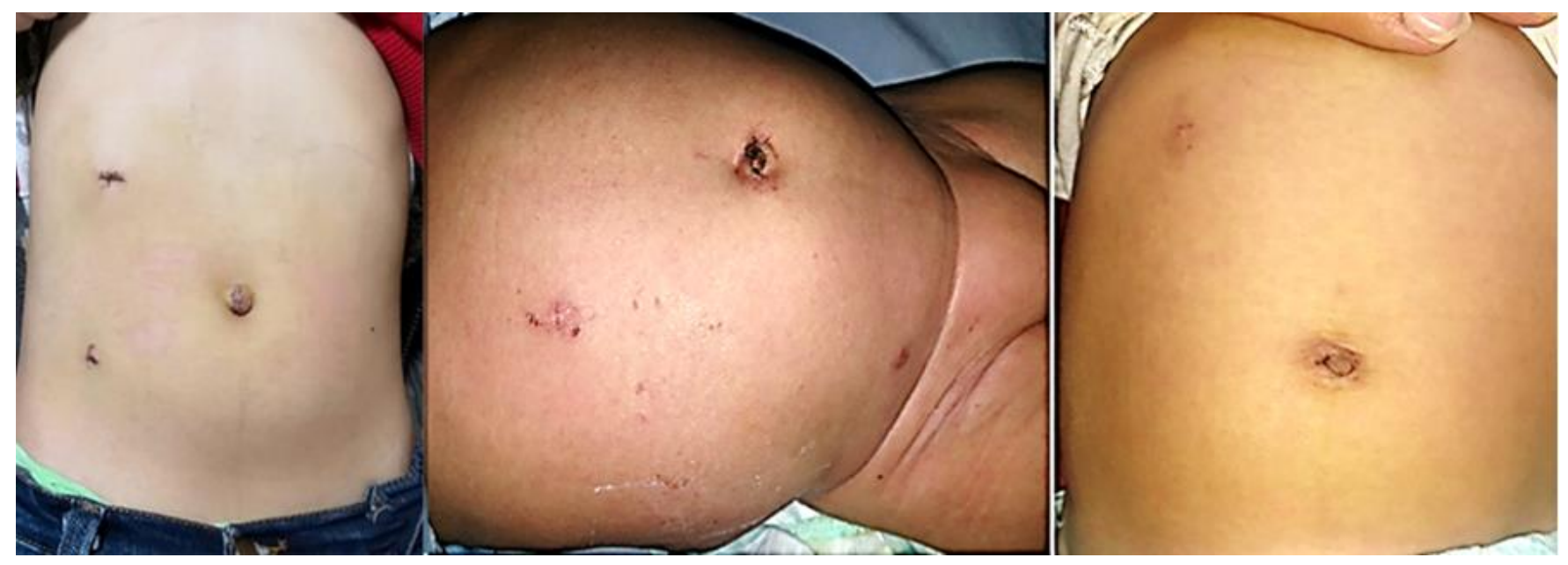

Figure 4: The postoperative cosmetic results after LP.

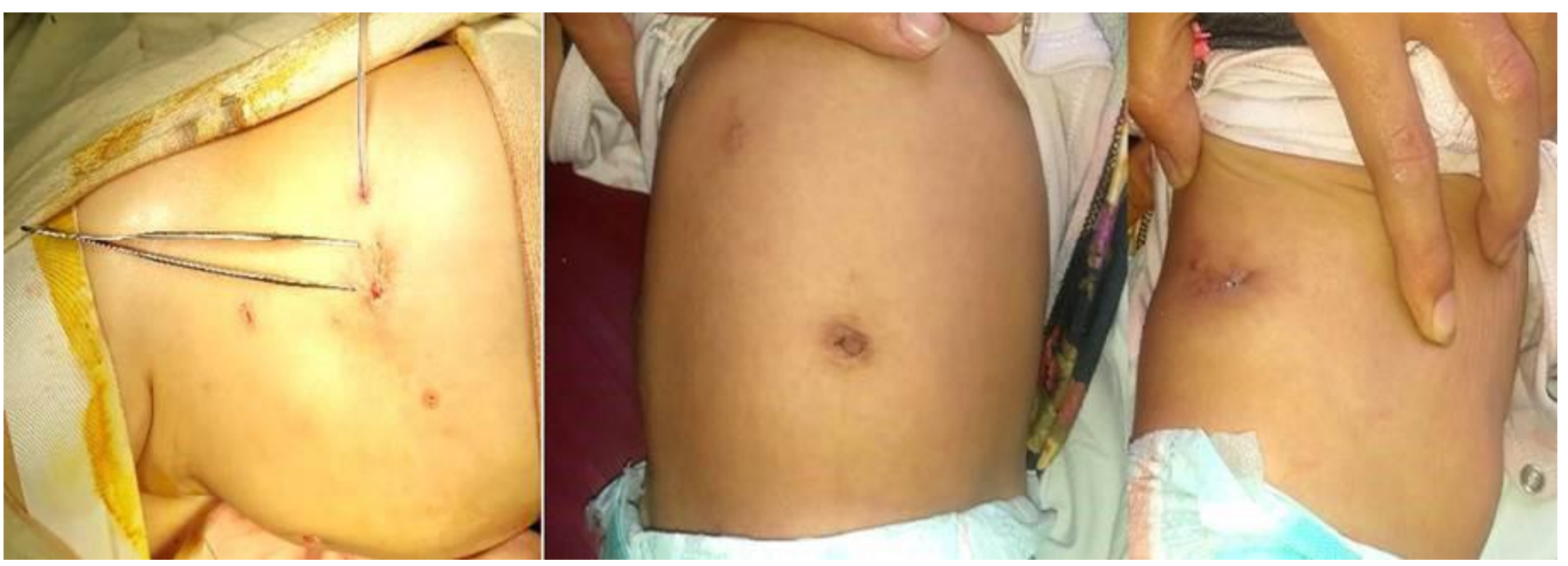

Figure 5: The postoperative cosmetic results after LAP. 
Table 2: intra- and postoperative measurements in both groups.

\begin{tabular}{|c|c|c|c|c|c|c|c|c|c|}
\hline & \multicolumn{8}{|c|}{ Groups } & \multirow{3}{*}{ P-value } \\
\hline & \multicolumn{4}{|c|}{ Group A No=10 } & \multicolumn{4}{|c|}{ Group B No=10 } & \\
\hline & Mean & Std & Number & $\%$ & Mean & Std & Number & $\%$ & \\
\hline Operative time in minutes & 154 & 21 & & & 114 & 12 & & & $0.001 *$ \\
\hline Blood loss in ml. & 88.8 & 19.3 & & & 54 & 13.5 & & & $0.001 *$ \\
\hline Additional port & & & 2 & $20 \%$ & & & 1 & $10 \%$ & $>0.05$ \\
\hline GIT recovery in hours & 9 & 3 & & & 9 & 3 & & & 1 \\
\hline Drainage amount in $\mathrm{ml}$ & 206 & 88 & & & 53 & 47 & & & $0.001 *$ \\
\hline Drain removal day & 5 & 2 & & & 4 & 1 & & & $0.001 *$ \\
\hline Analgesia free day & 6 & 2 & & & 8 & 1 & & & $0.001 *$ \\
\hline Hospital stay in days & 7 & 2 & & & 8 & 2 & & & 0.02 \\
\hline
\end{tabular}

* P-value less than 0.01 is highly significant.

Table 3: The anatomical improvement in hydronephrosis using SFU grading system in each group.

\begin{tabular}{|c|c|c|c|c|c|c|c|c|c|c|c|c|}
\hline & \multicolumn{10}{|c|}{ SFU Score } & \multirow{3}{*}{ P-value } \\
\hline & & \multicolumn{2}{|c|}{ Grade 0} & \multicolumn{2}{|c|}{ Grade 1} & \multicolumn{2}{|c|}{ Grade 2} & \multicolumn{2}{|c|}{ Grade 3} & \multicolumn{2}{|c|}{ Grade 4} & \\
\hline & & $\mathbf{N}$ & $\%$ & $\mathbf{N}$ & $\%$ & $\mathbf{N}$ & $\%$ & $\mathbf{N}$ & $\%$ & $\mathbf{N}$ & $\%$ & \\
\hline \multirow{2}{*}{ Preoperative } & Group A No=10 & & & & & & & 2 & $20 \%$ & 8 & $80 \%$ & \multirow{2}{*}{$>0.05$} \\
\hline & Group B No=10 & & & & & & & 3 & $30 \%$ & 7 & $70 \%$ & \\
\hline \multirow{2}{*}{ Postoperative } & Group A No $=10$ & & & & & 5 & $50 \%$ & 4 & $40 \%$ & 1 & $10 \%$ & \multirow{2}{*}{$>0.05$} \\
\hline & Group B No=10 & & & 4 & $40 \%$ & 5 & $50 \%$ & 1 & $10 \%$ & & & \\
\hline
\end{tabular}

Table 4: The functional improvement suing diuretic pyelogram in each group.

\begin{tabular}{|c|c|c|c|c|c|}
\hline & \multicolumn{4}{|c|}{ Groups } & \multirow{3}{*}{ P-value } \\
\hline & \multicolumn{2}{|c|}{ Group A No=10 } & \multicolumn{2}{|c|}{ Group B No=10 } & \\
\hline & Mean & Std & Mean & Std & \\
\hline Preoperative Split Function & 25.8 & 5.3 & 25.2 & 8.7 & \multirow{3}{*}{$0.001 *$} \\
\hline Postoperative Split Function & 40.2 & 18.7 & 41.7 & 13.3 & \\
\hline Degree of improvement & 24.9 & 18.2 & 26.5 & 13 & \\
\hline
\end{tabular}

* P-value less than 0.01 is highly significant.

\section{DISCUSSION}

Application of laparoscopy in pediatric urology has launched over more than 30 years coming from a merely diagnostic use for non-palpable testes to "interventional" laparoscopy and finally to the era of the reconstructive pediatric laparoscopic urology when Peters described the first laparoscopic pyeloplasty in a child in $1995^{(4)}$. Laparoscopic surgery in pediatric urology became implemented increasingly in the twenty-first century with now present-day applications including the complete variety of all kind of indications for surgery for pediatric urological pathology ${ }^{(12)}$.

Transperitoneal LP was the first approach described in the literature for minimally invasive surgery of UPJO in the pediatric population; however, it is still challenging and is primarily available at centers with advanced laparoscopic experience ${ }^{(13)}$. As the field continues to evolve with improvement in the laparoscopic techniques a new alternative (LAP) has been emerged in recent years to make the procedure easier and faster by sewing the anastomosis outside the abdomen ${ }^{(14)}$.

Since there have been few studies directly comparing recent surgical techniques ${ }^{(5)}$, in the present study, we described the clinical characteristics and compared the surgical outcomes of LP and LAP for the treatment of UPJO in children.

The mean age, sex distribution, laterality and presentation in the current series goes in parallel with that were mentioned in the literature. The predominance of the abdominal pain in the LP group can be explained by the older age of this group than the LAP group. Braga et al. mentioned that the lately diagnosed children with UPJO are mainly suffering from abdominal pain due to the presence of a ureteric kinking and adhesions in lately diagnosed children.

The delay in diagnosing those patients may be also attributed to low awareness of Dietl's crisis, first reported by Josef Dietl in 1864 which is described as episodic, crampy upper abdominal pain, nausea, and vomiting associated with intermittent renal pelvic 
obstruction $^{(15)}$. This study, as well as other studies, insisted on the high sensitivity of ultrasound to diagnose the UPJO during pregnancy leading to a discovery of around $60 \%$ of the cases prenatally ${ }^{(16)}$.

Unlike LAP, there were many studies recorded the average LP operative time. Juliano et al. ${ }^{(17)}$, for example, reported a mean operative time of $127 \mathrm{~min}$ [range $=45-370 \mathrm{~min}]$. The significant difference in the operative time found in several studies may be attributed to the presence of different surgeons with different experiences. The technique of suturing, the methods used for knot tying, the inclusion of recurrent UPJO, and the occurrence of intraoperative complications are important factors related to the operative time ${ }^{(18)}$.

In this study, the mean operative time in the LP group was slightly longer than Elemam's 143.9 $\min ^{(18)}$ and comparable to that was reported by others ${ }^{(19)}$. In the work of Mandhani et al., the mean operative time was $246 \mathrm{~min}$ which is much longer than ours ${ }^{(20)}$.

By comparison, the mean operative time in the LAP group was shorter than the LP group. This could be related to the learning curve of laparoscopic surgery and the fast hand-made anastomosis in the LAP group.

According to Glybochko et al. ${ }^{(21)}$, the use of 'familiar' instruments in a 'familiar' extracorporeal setting during upper urinary tract reconstructive surgery in patients with hydronephrosis improves the precision of the pyeloplasty procedure. Extracorporeal ureteral handling reduces surgery duration especially for surgeons with little experience in laparoscopic reconstructive surgery.

In our study, as well as in other studies ${ }^{(22)}$, the incidence of intraoperative blood loss was minimal and none of the patients needed a blood transfusion in both groups. The slight increase in blood loss in the LP group than the LAP group may be attributed to the longer operative time and the older age of patients than in the LAP group.

Ideally either the LP or the LAP needs 2 ports besides the camera port; however, sometimes surgeons need to insert a fourth port. Unlike LAP, this happened in the current study twice in the LP group. This could be attributed to the difficulty in manipulating the dilated renal pelvis through two instruments and might also due to the unfamiliarity of the operating surgeon with the technique as well.

Lasmar et al. reported a postoperative complication rate of $10.9 \%$ in 10 patients in the form of urine leakage (six cases), urinary fistula (one case), and port site infection (three cases), and all were managed conservatively ${ }^{(19)}$. Juliano et al. reported a $9.6 \%$ postoperative complication rate, and urine leakage occurred in eight cases (6.1\%); all cases were managed conservatively ${ }^{(17)}$.

Many authors found that LP was very difficult in certain situations, such as the recurrent pyeloplasty and intrarenal pelvis ${ }^{(18)}$. In the current study, there was a solitary case (10\%) in the LP group, who had no obvious renal pelvis dilatation by laparoscopy since the pelvis was completely intrarenal, needed conversion to open surgery since it had an intrarenal pelvis. By contrast, in the LAP group, there was no need to convert to open surgery in all the cases.

In this study, unlike the LAP group, $10 \%$ developed urine leakage in the LP group, which stopped spontaneously within a week. This matches Shoma et al.'s results who reported two cases of postoperative complications: one had mild hematuria, which was managed conservatively, and the second developed urine leakage, which stopped on the ninth postoperative day ${ }^{(23)}$. Wound complications were evident in the LAP group than the LP group where $20 \%$ suffered from wound problems, a half of them had a seroma under the wound and it was aspirated twice before complete resolution and the remaining $10 \%$ had an infection in the wound that was treated by continuous dressing and giving antibiotics according to culture and sensitivity tests.

Many authors like Bilen et al. ${ }^{(24)}$ mentioned that the mean hospital stay after LP ranges between 2.4 and 3.7 days. However, in the current study the LP patients' mean hospital stay was 7 days \pm 2 , compared to 8 days \pm 2 in the LAP group. Although the LP group left the hospital a day earlier than the LAP group, we still had a relatively longer hospital stay than the mentioned studies since most of our patients came from far places and many surgeons also insisted on staying of drains for a long time in order to be sure that there is no leak.

In this study, all patients started oral intake on the first day safely. The first postoperative day showed no significant difference between both groups; however, by the 3rd day, the pain was significantly improved in the LP group while the LAP group recorded higher pain scores.

The higher need for analgesia combinations and the higher score of pain in the LAP group than the LP group indicated that LP is better than LAP in terms of pain, analgesia, and even medications withdrawal. This can be explained by the shorter incisions and muscle cutting in the LP compared to the LAP.

Although both groups had preoperative SFU grade between 3 and 4 with a mean of 3.7, postoperatively, there was a good response in the LP group where $50 \%$ of patients improved to grade 2 and $40 \%$ had grade 3 and only one case $(10 \%)$ showed no anatomical improvement at grade 4. Compared to the LP group, the LAP group recorded a 
statistically insignificant better improvement in terms of anatomical recovery of hydronephrosis where $50 \%$ of patients settled at grade 2, 40\% allocated at grade 1 and the remaining $10 \%$ showed no marked improvement (grade 3).

In the LP group, the mean split function was $25.8 \% \pm 5.3$ preoperatively which was improved to $40.2 \% \pm 18.7$ with an improvement of $24.9 \pm 18.2$. Similarly, in the LAP group, the mean split function was $25.2 \% \pm 8.7$ preoperatively which was improved to $41.7 \% \pm 13.3$ with an improvement of $26.5 \pm 13$.

Based on the changes in the pre- and postoperative symptoms, MAG3 renogram and its washout curves and, US follow up, both groups achieved a similar high success rate of $90 \%$ in terms of both the anatomical and functional improvement in the current study with a recurrence rate of $10 \%$. The recurrent cases suffered from a recurrence of the flank pain, confirmed by radiological evidence of obstruction at the new UPJ.

In terms of cosmetic satisfaction, in the LP group, $80 \%$ of patients rated score 5, while the remaining $20 \%$ rated score 4 . By comparison, $40 \%$ of patients rated score 4, 40\% rated score 3 and $20 \%$ rated score 2 . This indicates that there was a great difference in parent's satisfaction in favor of LP.

As shown above, the current study matched many other studies searching the field of minimally invasive pyeloplasty in terms of the demographic and clinical characteristics which helped the current study's comparisons to concentrate on its main aims in terms of the operative time, the functional and anatomical outcomes and the cosmetic appearance between LP and LAP. The LP had the upper hand in cosmetic appearance however this privilege does not come at no cost. The LAP was much faster in the technique, easier in the performance and more suitable to the less experienced surgeons, providing the best choice for surgeons who want to maintain the balance between an acceptable cosmetic appearance along with easier and faster minimally invasive technique, and it could be considered as an important step in the ladder of mastering LP.

\section{CONCLUSION}

In the light of the current study, the main advantages of the LAP are the marked operative time reduction and its feasibility and reproducibility for the less experienced laparoscopic surgeons; however, this comes at the cost of the patient's cosmetic satisfaction and pain relief provided by the LP.

\section{LIMITATIONS}

Although this study was carefully prepared, we are still aware of its limitations and shortcomings. Limitations of the current study include the selection bias due to being done in different centers by different surgeons and the small number of the patients. Despite these limitations, our study addresses an important issue, which is seldom be addressed in the literature.

\section{REFERENCES}

1. Slojewski M (2014): Laparoscopic pyeloplasty with concomitant pyelolithotomy - too much of a good thing?, Cent European J Urol., 66(4): 445.

2. Samarasekera D, Chew B (2011): Endopyelotomy still has an important role in the management of ureteropelvic junction obstruction, Can Urol Assoc J., 5(2): 134-6.

3. Rashad F, Emam A, Mikkawy S et al. (2016): Stentless versus stented laparoscopic pyeloplasty, A comparative study, New York Science, 9(12): 162-5.

4. Peters C, Schlussel R, Retik A (1995): Pediatric Laparoscopic Dismembered Pyeloplasty, Journal of Urology, 153(6): 1962-65.

5. Song H, Lee C, Jung J et al. (2017): A comparative study of pediatric open pyeloplasty, laparoscopyassisted extracorporeal pyeloplasty, and robot-assisted laparoscopic pyeloplasty, PLoS One, 12(4): 175-86.

6. Freilich D, Penna J, Nelson P et al. (2010): Parental satisfaction after open versus robot assisted laparoscopic pyeloplasty: results from modified Glasgow Children's Benefit Inventory Survey, J Urol., 183(2): 704-8.

7. Sorensen D, Delostrinos C, Johnson H et al. (2011): Comparison of the learning curve and outcomes of robotic assisted pediatric pyeloplasty, J Urol., 185(6): 2517-22.

8. Autorino R, Eden C, El-Ghoneimi A et al. (2014): Robot-assisted and laparoscopic repair of ureteropelvic junction obstruction: a systematic review and metaanalysis, Eur Urol., 65(2): 430-52.

9. Fernbach S, Maizels M , Conway J (1993): Ultrasound grading of hydronephrosis: introduction to the system used by the Society for Fetal Urology, Pediatr Radiol., 23(6): 478-80.

10. Drendel A, Kelly B , Ali S (2011): Pain assessment for children: overcoming challenges and optimizing care, Pediatr Emerg Care, 27(8): 773-81.

11. Vargas-Schaffer $\mathbf{G}$ (2010): Is the WHO analgesic ladder still valid? Twenty-four years of experience, Canadian Family Physician, 56: 514-517.

12. Szavay P (2019): Applications of Laparoscopic Transperitoneal Surgery of the Pediatric Urinary Tract, Front Pediatr., 7: 124-129.

13. Sweeney D , Docimo S (2011): Pediatric Laparoscopic (Infant, Pre-pubertal, and Teenager) Pyeloplasty for Ureteropelvic Junction Obstruction in Robotic and Laparoscopic Reconstructive Surgery in Children and Adults (Ost M, ed): 93-108, Humana Press, Totowa, New Jersey (US).

14. Sharifabad $P$, Hiradfar M, Shojaeian $R$ et al. (2018): Laparoscopic-Assisted Extracorporeal Pyeloplasty: A Minimally Invasive Approach to Pediatric Ureteropelvic Junction Obstruction, NephroUrology Monthly, 10(5): e83823. 
15. Alagiri $M$, Polepalle $S$ (2006): Dietl's crisis: an under-recognized clinical entity in the pediatric population, Int Braz J Urol., 32(4): 451-3.

16. Braga L, Liard A, Bachy B et al. (2003): Ureteropelvic junction obstruction in children: two variants of the same congenital anomaly?, Int Braz J Urol., 29(6): 528-34.

17. Juliano R, Mendonca R, Meyer F et al. (2011): Long-term outcome of laparoscopic pyeloplasty: multicentric comparative study of techniques and accesses, J Laparoendosc Adv Surg Tech A., 21(5): 399-403.

18. Elemam A, Shalaby R, Ismail M et al. (2015): Transperitoneal laparoscopic pyeloplasty in children and adolescents, Annals of Pediatric Surgery, 11(4): 231-238.

19. Lasmar M, Castro H, Vengjer A et al. (2010): Transperitoneal laparoscopic pyeloplasty: Brazilian initial experience with 55 cases, Int Braz J Urol., 36(6): 678-84.

20. Mandhani A, Kumar D, Kumar A et al. (2005): Safety profile and complications of transperitoneal laparoscopic pyeloplasty: a critical analysis, J Endourol., 19(7): 797-802.

21. Glybochko P, Alyaev Y, Grigoryan V et al. (2016): Extracorporeal ureteral handling during laparoscopic pyeloplasty, European Urology Supplements, 15(6): 143.

22. Inagaki T, Rha K, Ong A et al. (2005): Laparoscopic pyeloplasty: current status, BJU Int., 95 (2): 102-5.

23. Shoma A, El Nahas A, Bazeed M (2007): Laparoscopic pyeloplasty: a prospective randomized comparison between the transperitoneal approach and retroperitoneoscopy, J Urol., 178(5): 2020-4.

24. Bilen C, Bayazit Y, Gudeloglu A et al. (2011): Laparoscopic pyeloplasty in adults: stented versus stentless, J Endourol., 25(4): 645-50. 